EPJ Web of Conferences 71, 00138 (2014)

DOI: $10.1051 /$ epjconf/20147100138

(C) Owned by the authors, published by EDP Sciences, 2014

\title{
Highlights from the Daya Bay Neutrino Experiment
}

\author{
Zhe Wang $^{1, \mathrm{a}}$ for the Daya Bay Collaboration \\ ${ }^{1}$ Department of Engineering Physics, Tsinghua University, Beijing, China
}

\begin{abstract}
With an understanding of the energy response of the anti-neutrino detectors, the Daya Bay collaboration presents new results using gadolinium-neutron capture: $\sin ^{2} 2 \theta_{13}=0.108 \pm 0.028$ and $\left|\Delta m_{e e}^{2}\right|=2.55_{-0.18}^{+0.21} \times 10^{-3} \mathrm{eV}^{2}$ with only the distortion information of the neutrino energy spectrum shape, and $\sin ^{2} 2 \theta_{13}=0.090_{-0.009}^{+0.008}$ and $\left|\Delta m_{e e}^{2}\right|=2.59_{-0.20}^{+0.19} \times 10^{-3} \mathrm{eV}^{2}$ with both the shape and event rate information. It is also demonstrated that a clean inverse beta decay sample can be extracted using hydrogenneutron capture, which is now being used for neutrino oscillation measurement. The supernova online trigger is designed and implemented, which can provide about $100 \%$ efficiency for all SN1987A-scale supernova bursts within the Milky Way.
\end{abstract}

\section{Introduction}

The Daya Bay Experiment studies reactor electron-antineutrino disappearance by measuring the inverse beta decay (IBD) event ratio between near and far sites $[1,2]$. With more statistics and a better understanding of the systematics, improvement in the precision of $\sin ^{2} 2 \theta_{13}$ can be expected, which is critical for the next milestone of neutrino oscillation study, discovering leptonic CP violation [3]. Here only the most recent progress is introduced, including the results of reactor neutrino oscillation analysis with the delayed neutron captured on gadolinium $(\mathrm{nGd})$, the status of the analysis with neutron captured on hydrogen $(\mathrm{nH})$ and the feature of the supernova trigger.

\section{Energy response and $n G d$ shape analysis}

The energy response of the antineutrino detectors (ADs) is studied by 1) various constant-energy gamma sources, including ${ }^{68} \mathrm{Ge},{ }^{60} \mathrm{Co}$ and ${ }^{241} \mathrm{Am}-{ }^{13} \mathrm{C}$, which are employed regularly, ${ }^{137} \mathrm{Cs},{ }^{54} \mathrm{Mn}$, ${ }^{40} \mathrm{~K},{ }^{241} \mathrm{Am}-{ }^{9} \mathrm{Be}$ and $\mathrm{Pu}-{ }^{13} \mathrm{C}$ which are deployed during special calibration periods, natural radioactivities inside the detector, ${ }^{40} \mathrm{~K},{ }^{208} \mathrm{Tl}$, and neutron capture on $\mathrm{H}$, and 2) the continuous beta decay spectrum from muon spallation product ${ }^{12} \mathrm{~B}$.

The best oscillation analysis result is shown in Fig.1. The analysis with only the distortion information of the neutrino energy spectrum shape gives $\sin ^{2} 2 \theta_{13}=0.108 \pm 0.028$ and $\left|\Delta m_{e e}^{2}\right|=2.55_{-0.18}^{+0.21} \times$ $10^{-3} \mathrm{eV}^{2}$, and the rate plus shape analysis gives $\sin ^{2} 2 \theta_{13}=0.090_{-0.009}^{+0.008}$ and $\left|\Delta m_{e e}^{2}\right|=2.59_{-0.20}^{+0.19} \times 10^{-3}$ $\mathrm{eV}^{2}$. More details can be found in [4].

\footnotetext{
a e-mail: wangzhe-hep@tsinghua.edu.cn
} 


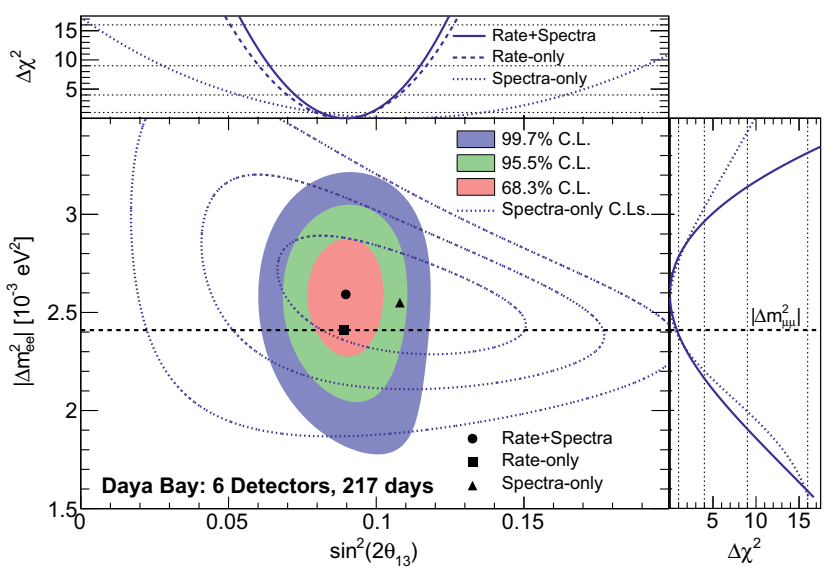

Figure 1. (Color online) Allowed regions for the neutrino oscillation parameters $\sin ^{2} 2 \theta_{13}$ and $\left|\Delta m_{e e}^{2}\right|$ at the 68.3, 95.5 and $99.7 \%$ confidence levels, obtained from comparison of the rates and prompt energy spectra measured by the 3 near-site and 3 far-site antineutrino detectors (solid regions). The best estimate of the oscillation parameters is given by the black dot. The three dotted contours indicate the allowed 68.3, 95.5 and 99.7\% C.L. regions for the spectra-only fit with the black triangle representing best estimate of the oscillation parameters. The adjoining panels show the dependence of $\Delta \chi^{2}$ on $\left|\Delta m_{e e}^{2}\right|$ (right) and $\sin ^{2} 2 \theta_{13}$ (top). The black square and dashed curve represent the rate-only result. The dotted curves represent the spectra-only $\Delta \chi^{2}$ distributions. The dashed horizontal line represents the MINOS $\left|\Delta m_{\mu \mu}^{2}\right|$ measurement.

\section{$3 \mathrm{nH}$ analysis}

With also considering neutron capture on hydrogen, the statistics of IBD candidates at the Daya Bay is doubled and thus enabling us an improvement of the overall sensitivity of the experiment and a deeper understanding about the Daya Bay detectors and reactors. However, through conservative estimations, Gd capture was believed to be the only way to reach sub-percent precision and so the detector was optimized for this purpose. The high accidental background due to the low-energy delayed signal, only $2.2 \mathrm{MeV}$, and long capture time, $200 \mu \mathrm{s}$, and the more complicated detector systematic uncertainty make the study very challenging.

Using random single events selected from data and the normalization factor proposed in [5], the accidental background scale and shape are precisely predicted. The systematic uncertainty is verified using coincidence candidates with vertex separations larger than $2 \mathrm{~m}$, see Fig.2. After accidental background subtraction, their differential distribution and total number of entries agree well with zero.

As explained above the distance distribution can be used to verify the accidental background prediction. Therefore, it also has the power to reject accidentals. Since the signal can be directly observed after the accidental background subtraction, the distance cut efficiency, as well as the identicalness between ADs, can be directly measured with data.

After applying a $0.5 \mathrm{~m}$ cut for the distance and subtracting the accidental background, the prompt vs. delayed energy distribution for the near and far sites is shown in Fig. 3. Clear $\mathrm{nH}$ and $\mathrm{nGd}$ signals are both seen. 


\section{ICNFP 2013}

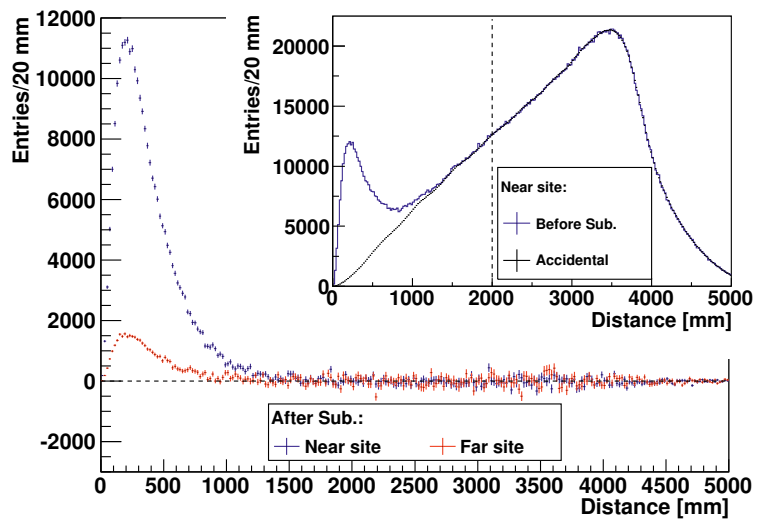

Figure 2. (Color online) (Preliminary) Distributions of the distance between the prompt and the delayed vertices after the accidental backgrounds are subtracted for the near sites (blue lines) and the far site (red lines), respectively. The inset plot shows the distance distributions for both the selected coincidence events (blue lines) and the predicted accidental background (black lines). Real correlated events populated at $0.2 \mathrm{~m}$ and the long tail beyond $2 \mathrm{~m}$ after the accidental subtraction agrees well with zero.

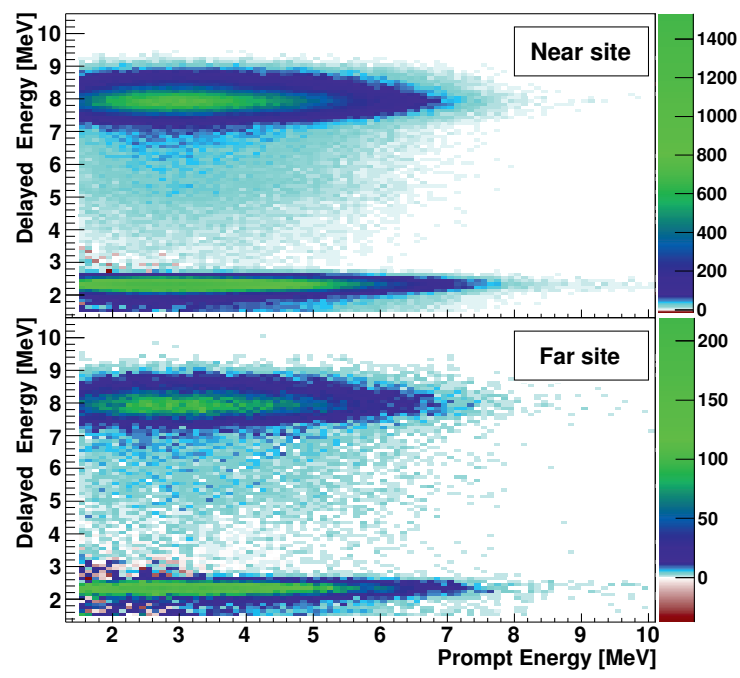

Figure 3. (Color online) (Preliminary) The prompt vs. delayed energy distribution after accidental background subtraction for the near (top) and far (bottom) sites. Clean $\mathrm{nH}$ and nGd signals can be observed. 


\section{Supernova trigger}

Online IBD event rates are monitored for an instantaneous increase within $10 \mathrm{~s}$ among all ADs as a trigger of supernova burst [6]. The major background is from fast neutrons caused by cosmic-ray muons and real IBD events from reactor neutrinos. But with eight ADs at three sites which are around $1 \mathrm{~km}$ apart from each other, the supernova trigger reaches about $100 \%$ efficiency for all 1987A-scale supernova bursts within the Milky Way, while maintaining a false alarm rate of $1 /$ year.

\section{References}

[1] F. P. An et al. (Daya Bay collaboration), Phys. Rev. Lett. 108, 171803 (2012).

[2] F. P. An et al. (Daya Bay collaboration), Chinese Phys. C 37, 011001 (2013).

[3] C. Adams et al. (LBNE collaboration), arXiv:1307.7335 (2013).

[4] F. P. An et al. (Daya Bay collaboration), arXiv:1310.6732 (2013).

[5] Jingyi Yu et al. arXiv:1301.5085 (2013).

[6] Hanyu Wei et al. arXiv:1310.5783 (2013). 